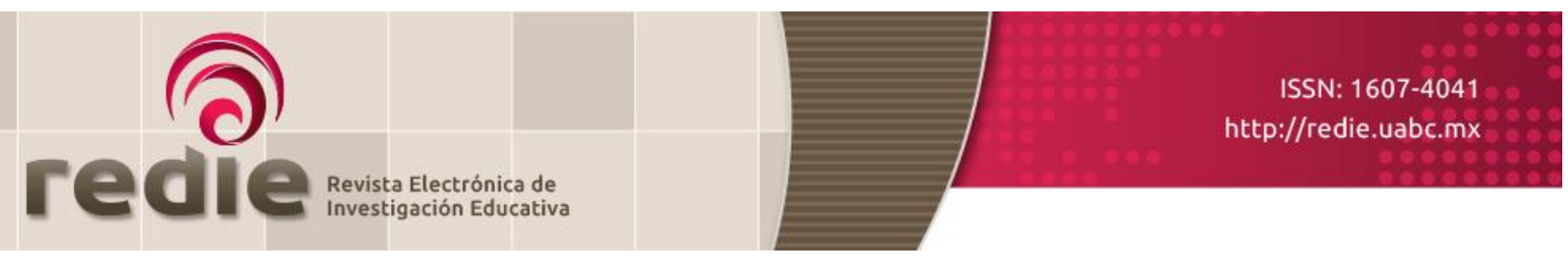

Vol. 20, Núm. 1, 2018

\title{
Violencia escolar: la perspectiva de estudiantes y docentes
}

\section{School Violence: The Perspective of Students and Teachers}

\author{
Berenice Pacheco-Salazar (*) bereniceps@gmail.com \\ (*) Universidad de Sevilla \\ (Recibido: 9 de agosto de 2016; Aceptado para su publicación: 3 de noviembre de 2016)
}

Cómo citar: Pacheco-Salazar, B. (2018). Violencia escolar: la perspectiva de estudiantes y docentes. Revista Electrónica de Investigación Educativa, 20(1), 112-121. https://doi.org/10.24320/redie.2018.20.1.1523

\section{Resumen}

El objetivo de la investigación realizada fue conocer las concepciones que tienen estudiantes y docentes sobre las causas de la violencia escolar. Se realizó un estudio descriptivo con diseño cualitativo en dos centros educativos del segundo ciclo de la enseñanza primaria en República Dominicana, en una población de 604 estudiantes y 23 docentes. El levantamiento de información se realizó a través de talleres lúdico-creativos, entrevistas a profundidad, grupos focales y observación no participante. Los resultados de la investigación destacan la existencia de débiles lazos de amistad, apego a los estereotipos de género e interiorización de la violencia como una forma de diversión. Las conclusiones son relevantes para el diseño e implementación de programas de atención y erradicación de la violencia escolar.

Palabras clave:Violencia escolar, convivencia escolar, relaciones entre pares, educación primaria.

\section{Abstract}

The objective of the research was to understand the perceptions held by students and teachers regarding the causes of school violence. A descriptive, qualitative study was performed in two second-cycle elementary education establishments in the Dominican Republic, with a population of 604 students and 23 teachers. Information was collected through playful, creative workshops; in-depth interviews; focus groups; and non-participant observation. The study results highlight weak friendships, adherence to gender stereotypes, and internalization of violence as a form of entertainment. The conclusions are relevant for the design and implementation of assistance programs and the eradication of school violence.

Keywords: Violence, school coexistence, peer relationships, primary education.

\section{Introducción}

La violencia está basada en la desigualdad y el abuso de poder. Es un comportamiento culturalmente aprendido que implica una direccionalidad y una intencionalidad de controlar, imponer, manipular o dañar a otros (Díaz-Aguado, 2007; Garaigordobil y Oñederra, 2010; Ortega, 2008; Torrego, 2006). En el ámbito 
escolar, la violencia puede ser entendida como cualquier acción que ocurra y se geste en la escuela, dificultando su finalidad educativa y lesionando la integridad de algún miembro de la comunidad escolar. En palabras de Ortega (2008), es un modelo de relación desigual entre aquellos de los que se espera una relación igualitaria.

Más allá de ser un fenómeno esporádico o episódico, la violencia escolar es un problema sistémico que afecta las dinámicas sociales sobre las que debe producirse la actividad educativa y, por tanto, constituye un obstáculo para la mejora de la calidad educativa, el logro de los aprendizajes y el sano desarrollo de estudiantes y docentes (Abramovay, 2005; Díaz-Aguado, 2007; Perrenoud, 2008).

En República Dominicana, estudios previos señalan que el uso de la violencia está culturalmente aceptado como mecanismo disciplinario en las escuelas (Vargas, 2010), y que la cantidad de víctimas y agresores en las escuelas dominicanas se sitúa por encima de la media de otros países de América Latina y de España, sobre todo en lo referido a robo, amenazas, rechazo y violencia sexual (Fundación Santa María y Organización de Estados Iberoamericanos, 2008).

Una investigación más reciente (Instituto Dominicano de Evaluación e Investigación de la Calidad Educativa [IDEICE] y Universidad Iberoamericana, 2014), que trabajó con una muestra de 2,391 escolares, reveló que la tipología de violencia más común en el contexto escolar ocurre entre estudiantes, sobre todo manifestada en gritos e insultos (24.1\%), golpes (13.6\%) y amenazas o intimidación (7.1\%). Un 69.1\% del estudiantado reportó haber presenciado violencia entre pares y un 33.6\% haber sido acosado en los últimos 2 meses.

La violencia escolar debe ser entendida desde el contexto social en el que tiene lugar (Debarbieux y Blaya, 2010; Viguer y Solé, 2012). En este sentido, la construcción social de la masculinidad y la feminidad se constituye en uno de los principales soportes de la violencia escolar, en tanto existe una inseparabilidad entre el estereotipo masculino tradicional y las actitudes violentas y discriminatorias.

Díaz-Aguado (2005) y Díaz-Aguado y Martínez (2014) plantean tres principales similitudes entre la violencia escolar y la de género: el perfil de quienes acosan, la tendencia a culpar a la víctima y las características de la escuela tradicional que obstaculizan la erradicación de la violencia. Además, se ha establecido que el arraigo a los estereotipos tradicionales de género y la interiorización de que la violencia es parte intrínseca de la identidad masculina, son aspectos que contribuyen a gestar un entorno escolar y social que propicia, tolera y justifica el uso de la violencia.

Conocer las formas de comprender y experimentar la violencia escolar que tienen estudiantes y docentes, así como sus percepciones sobre las causas que la originan y reproducen, se constituye en un punto de partida clave en el diseño e implementación de programas para la atención y prevención de la violencia escolar. El objetivo de esta investigación fue conocer las concepciones sobre las causas de la violencia escolar que tienen estudiantes y docentes, del segundo ciclo del nivel primario en República Dominicana.

\section{Metodología}

La investigación se llevó a cabo en dos centros educativos públicos del segundo ciclo del nivel primario, de contextos urbanos marginales en Santo Domingo, República Dominicana. Los mismos se seleccionaron entre aquellos que compartían condiciones similares de ubicación geográfica y vulnerabilidad económica.

El enfoque metodológico del estudio es cualitativo, de corte descriptivo y no experimental. Se trabajó con una muestra intencionada de 23 docentes y 604 estudiantes de 5o. a 80. grado, con edades comprendidas entre los 9 y 16 años. Tanto estudiantes como docentes participaron del estudio de manera informada y voluntaria.

Para el levantamiento y análisis de la información se utilizaron procedimientos propuestos por la teoría fundamentada (Strauss y Corbin, 2002; Giraldo, 2011; Restrepo-Ochoa, 2013). Para profundizar en las opiniones sobre las causas de la violencia escolar se utilizaron cuatro técnicas de recolección de 
información: observación no participante, talleres lúdico-creativos, grupos focales y entrevistas a profundidad semiestructuradas. El trabajo de campo se desarrolló durante el período comprendido de febrero a diciembre de 2015, implicando una inmersión de cuatro meses en cada uno de los centros educativos.

Tabla I. Síntesis del trabajo de campo realizado

\begin{tabular}{ll}
\hline Técnicas utilizadas & Datos levantados \\
\hline Observaciones no-participantes & $\begin{array}{l}165 \text { horas de observación registradas en diarios de campo, } \\
\text { utilizando un sistema descriptivo-narrativo abierto. }\end{array}$ \\
\hline Talleres lúdico-creativos & 12 talleres con participación de 300 estudiantes. \\
\hline Grupos focales & 45 grupos focales con 304 estudiantes. \\
\hline Entrevistas a profundidad & $\begin{array}{l}23 \text { entrevistas con igual número de personal docente } \\
\text { y de gestión pedagógica. }\end{array}$ \\
\hline
\end{tabular}

En la metodología empleada destaca como aspecto innovador el diseño e implementación de los talleres lúdico-creativos, en los que se trabajó con dinámicas de expresión teatral, dibujo y escritura creativa. Esto contribuyó a crear empatía y confianza con el estudiantado, que pudo expresarse espontáneamente sin el condicionamiento de categorías consideradas a priori.

En este estudio, el sistema de categorías para el análisis de la información fue desarrollado de manera emergente, siguiendo lo establecido por la teoría fundamentada. Todos los datos fueron analizados utilizando el programa informático MAXqda 12, los códigos asignados a las grandes categorías se especifican en la tabla II.

Tabla II. Principales categorías de análisis y códigos asignados

\begin{tabular}{|c|c|}
\hline \multirow{7}{*}{$\begin{array}{l}\text { CAU } \\
\text { Consideraciones sobre las causas } \\
\text { de la violencia escolar }\end{array}$} & CAu.com Causas vinculadas a contexto comunitario \\
\hline & CAu.FAm Causas vinculadas a contexto familiar \\
\hline & CAU.GEN Causas vinculadas a la construcción social de género \\
\hline & CAU.BIO Causas vinculadas a origen biológico \\
\hline & CAU.JuE Causas vinculadas al juego \\
\hline & CAu.Jer Causas vinculadas a la jerarquía social y abuso de poder \\
\hline & CAU.OTRO Otras causas \\
\hline
\end{tabular}

La triangulación de cuatro técnicas de levantamiento de información, la intensiva inmersión en el trabajo de campo, y la participación -junto con la investigadora- de tres investigadores externos en el proceso de codificación de los datos de ambos centros educativos, aportan validez y confiabilidad a la investigación.

\section{Presentación y discusión de resultados}

El cuerpo docente plantea que el contexto familiar constituye el principal causante de la violencia escolar, ya sea por la violencia intrafamiliar existente, por la reproducción de patrones de violencia que han aprendido, y/o por la falta de educación en valores.

Los niños generan esa violencia porque eso es lo que ven en la casa. (...) Explotan aquí con cosas que pasan en la casa, con violencia que ellos mismos sufren en la casa. (Coordinadora pedagógica).

El problema de los estudiantes radica en la familia. Yo diría, la violencia familiar y la ausencia de la tutoría paterna y la falta de valores. (Profesor, centro A).

Todo alrededor de ellos es violencia. Aprenden de lo que el papá le hace a la mamá y de los golpes que les dan a ellos mismos. Entonces desde pequeños son violentos porque eso es lo que ven. (Profesora, centro B). 
Se ha determinado que provenir de un contexto de violencia intrafamiliar es un factor de riesgo y predictivo del uso de la violencia (Milletich, Kelley, Doane y Pearson, 2010; Díaz-Aguado y Martínez, 2014; Giordano, Kaufman, Manning y Longmore, 2015; Gómez-Ortiz, Renner y Whitney, 2012; Romera y OrtegaRuiz, 2016); asimismo, que un entorno familiar caracterizado por el afecto, la comunicación y la promoción de la autonomía se constituye en un factor de protección (Gómez-Ortiz, Del Rey, Casas y Ortega, 2014). En ese sentido, estudios nacionales e internacionales señalan que el personal docente considera que una de las principales causas de la violencia escolar es el ambiente desestructurado y agresivo de las familias (Alonso, 2009; IDEICE e Ibero, 2014; Jares, 2006). No obstante, esta consideración es riesgosa pues puede convertirse en un mecanismo para desplazar responsabilidad e impedir que el centro educativo reflexione críticamente sobre su rol en la reproducción de la violencia.

Por su parte, el estudiantado también reconoce la influencia de las relaciones familiares en las situaciones de violencia que ocurren en sus centros.

A veces los hijos ven discutir a los padres y con los hermanos se dan muchos golpes, y vienen a la escuela así porque es como los han criado. (Alumno 5o., centro A).

A esos padres les gusta darles a los muchachos sin compasión, así ellos van creciendo y después quieren darles golpes a los demás. (Alumna 8o., centro B).

Sin embargo, los estudiantes ofrecen una explicación más amplia sobre la violencia escolar. Expresan que el juego y los estudiantes "enchinchadores", o espectadores provocadores, son los principales causantes de la violencia existente en los centros educativos. Además, sus opiniones ponen de manifiesto la consideración implícita de que los débiles lazos de amistad entre pares la sustentan.

\subsection{Débiles lazos de amistad}

El estudiantado de todos los grados de ambos centros educativos reportó la existencia de débiles vínculos de amistad con sus pares.

En el mundo no hay amigas. En esta escuela no hay amigas. (Alumna 60., centro B).

Ya nadie tiene amigos en estos tiempos. (Alumno 80., centro A).

En los talleres lúdico-creativos y en los grupos focales el estudiantado declaró tener enemistad con sus compañeros del mismo grado y aula, revelándose la noción de traición como una representación relevante de las relaciones entre pares. Los frágiles vínculos de amistad propician violencia y, a su vez, la violencia lesiona las relaciones de compañerismo generando desconfianza y desconexión.

Yo no tengo ninguna porque toditas son traicioneras. Todas son malas y si tú dices algo de ellas y de otras, ellas van y lo dicen. (Alumna 6o., centro A).

Todo el mundo traiciona. Yo aquí no tengo amigos. Ese que era mi amigo me robó y nos quedamos enemigos. (Alumno 60., centro B).

La amiga te hace un papel por delante y otro por atrás. Yo digo que ya no hay amigas. (Alumna 7o., centro B).

Los amigos te clavan un cuchillo por la espalda. Ya no hay amigos. Todo el mundo te traiciona. Yo tenía un amigo y mira lo que me hizo, me dio pila [muchos] de golpes. (Alumno 8o., centro A).

Estos hallazgos son relevantes, pues las relaciones interpersonales son consideradas un indicador de convivencia armónica y factor de protección ante la violencia escolar. Asimismo, se reconoce la intolerancia, la falta de solidaridad y los débiles lazos de amistad como obstaculizadores del clima escolar y factores de riesgo para la ocurrencia de violencia en dicho contexto (Córdoba, Del Rey, Casas y Ortega, 
2016; DeRosier y Newcity, 2005; Foshee, Benefield, McNaughton, Ennett, Faris et al., 2013; Murillo y Becerra, 2009; Vagi, Rothman, Latzman, Tharp, Hall y Breiding, 2013; Waldrip, Malcolm y JensenCampbell, 2008).

\subsection{Violencia como diversión}

Los estudiantes representan la violencia escolar como una forma de diversión, lo cual se evidencia en sus formas de jugar y utilizar su tiempo libre en el patio escolar. El trabajo de campo reveló la existencia de juegos que implican el ejercicio de violencia física o sexual.

Hay un juego que le dicen dizque "la puse" y cuando tú la pones mal [la mano] te entran a trompadas. (Alumno 6o. centro B).

Nosotros jugamos un juego que se llama a la patá' [dándose patadas]. (Alumno 7o., centro A).

(...) si tú pierdes entonces te entran a golpes todos en la cabeza. (Alumnos 7o., centro B).

R: Lo varones se le ponen atrás a las hembras.

R: Agarrándole el culo y meneándose.

R: Pegándosele atrás. (Alumnas 60., centro A).

R: Ella viene y te da un beso, y cuando son novios es con la lengua.

R: Y si no quieres besos, entonces aguantas un trompón. (Alumnos 70., centro A).

Además de jugar de manera violenta, los estudiantes consideran que los juegos son causa de la violencia escolar y que, a su vez, la violencia es consecuencia inherente a todo juego o broma. A su entender, parece no existir diferencia significativa entre jugar y ejercer violencia.

Uno comienza jugando y termina peleando. (Alumno 5o., centro A).

Del relajo [bromas] y los juegos es que viene la violencia, porque yo puedo estar relajando contigo y después empieza el problema. (Alumna 5o., centro B).

Siempre comienza por el juego y siempre acaba mal. Del juego y el relajo es que viene la violencia. (Alumna 6o., centro A).

Para mí todo lo que primero es juego después termina en violencia. Como el martes, ¿tú viste?, estaban dos relajando a las trompadas y salieron peleando de una vez. (Alumna 7o., centro A).

Yo creo que la violencia pasa cuando uno se propasa de la raya. Siempre los relajos y los juegos llevan a la violencia. (Alumna 8o., centro B).

En los talleres lúdico-creativos fue común que estudiantes utilizaran las mismas poses para representar la acción de jugar y de ejercer violencia: jalarse los cabellos, golpearse y empujarse, por ejemplo. De igual modo, las dramatizaciones de situaciones de violencia producían carcajadas entre ellos. Llama también la atención que muchas situaciones de violencia sexual de alumnos hacia alumnas son justificadas y minimizadas, pues se consideran relajo o juego.

El hallazgo de la violencia experimentada como una forma de diversión es coherente con estudios previos, que demuestran que el estudiantado consideran la violencia como un mecanismo de reconocimiento social, de satisfacción personal y como una experiencia agradable y divertida (Gómez, 2013; Kerbs y Jolley, 2007). Equiparar juego y violencia es una manera de normalizar esta última y 
garantizar su omnipresencia. Aprender a jugar de manera violenta e interiorizar que la violencia es un juego, genera desconexión emocional y moral.

Estudiantes provocadores y espectadores. Se reconoce la existencia de distintos roles en la violencia escolar: víctimas, agresores, espectadores, ayudantes de quien agrede y defensores de la víctima (Del Rey y Ortega, 2007; Díaz-Aguado, 2005).

En el presente estudio, otra evidencia de la representación de la violencia como forma de diversión se encuentra en que los estudiantes asumen el rol de espectadores ante episodios de violencia, los cuales parecerían incluso ser deseados como forma de entretenimiento y para interrumpir las clases.

Cuando se están fajando [peleando] uno se divierte mirando. (Alumno 5o., centro B).

Cuando una le da a la otra y así, da risa. Sí, claro que da risa. Cuando hay uno peleando uno goza con eso. (Alumna 6o., centro B).

Le dan a uno y se está riendo el que está al lado. (Alumno 7o., centro B).

A algunas gentes les gusta ver peleando a los otros. Se emocionan cuando ven una gente peleando. Comienzan a enchincharlo para ver el pleito. (Alumno 7o., centro A).

Los estudiantes plantean la existencia de pares "enchinchadores" (de chinchar, molestar) o, lo que es lo mismo, espectadores provocadores; es decir, estudiantes que no están directamente involucrados en los episodios de violencia, pero que incitan a que estos ocurran.

Para un pleito [pelea o discusión] tiene que haber otra persona, tres personas: la que enchincha y las otras dos que van a pelear. (Alumna 70., centro A).

Siempre hay uno que lo va a agitar y enchinchar para que usted pelee. (Alumno 7o., centro B).

Tú puedes estar peleándote y el amigo en vez de desapartarte lo que hace es que mejor empieza a enchincharte. (Alumno 80., centro A).

Según el estudiantado entrevistados, esparcir chismes y falsos rumores constituye el principal mecanismo utilizado por sus pares "enchinchadores" para que sucedan situaciones de violencia.

Y hay algunas chismosas que viven dando mandados aquí y allá, y así es que empieza todo, con los chismes. (Alumna 6o., centro A).

Todo empieza por los enchinchadores que mandan a tirar puyas [esparcir falsos rumores]. (Alumno 7o., centro A).

Muchas peleas empiezan por los chismes. Empiezan a decirle a uno cosas que dizque la otra dijo siendo mentira, sólo para enchinchar. Por eso es que digo que tienen que haber tres personas para surgir un pleito. (Alumna 7o., centro B).

Es posible afirmar que el acto de "enchinchar" es en sí mismo una manifestación de violencia verbal y que guarda estrecha relación con la concepción interiorizada de la violencia como un modo de diversión.

\subsection{Violencia justificada en la construcción social de género}

Docentes y estudiantes no reconocen la construcción desigual de género como causa de la violencia escolar, sin embargo, durante el trabajo de campo se manifestó el arraigo de ambos a los estereotipos tradicionales de género. Estudiantes y docentes de todos los grados evidenciaron una normalización del comportamiento violento como parte de la identidad masculina, lo que -a su vez- otorga a los alumnos 
mayor permisividad ante el ejercicio de la violencia.

Ellos tienden a ser más violentos que las hembras. Sí, porque son hombres se comportan así. (Profesora, centro B).

Los varones son así, violentos, atrevidos. (Alumna 5o., centro B).

Yo no me voy a quedar "dao" [golpeado]. El que me da yo le doy. Como hombre me tengo que dar a respetar. Al que me dé un mal golpe, yo le doy dos. (Alumno 7o., centro A).

Además, la internalización de los estereotipos de género también se hizo evidente a través de tres creencias o comportamientos:

a) Alumnas que se comportan desde los parámetros de la cultura patriarcal, asumiendo su cuerpo como objeto para el placer de otros.

Ella dijo en el baño: "yo me voy a poner los pantalones apretados para que los niños me enamoren". (Alumna 5o., centro B).

Hay una muchacha que deja que le agarren las tetas, que le agarren las nalgas, que le agarren todo. Usted sabe, así es que uno logra su conquista. (Alumna 5o., centro A).

b) Alumnos que minimizan y justifican episodios de violencia sexual considerándolos un juego o una broma.

A algunas les manoseamos las tetas en los baños, pero eso es un juego. (Alumno 7o., centro B).

Algunos le agarran las tetas, pero eso es un relajo. (Alumno 8o., centro A).

c) Docentes y estudiantes que justifican la violencia, sobre todo la sexual, de la que son víctimas las alumnas cuando exhiben un comportamiento considerado promiscuo, planteando que ellas así lo provocan y que, por tanto, son merecedoras de la misma. De esta manera, se legitima el abuso sexual, justificando al victimario y culpando a la víctima.

Ella es muy puta. Lo que le pasa es porque se lo busca. (Alumna 5o., centro A).

Cuando las muchachitas se ponen los pantalones así [apretados] y la camisa se la ponen así [mostrando el abdomen] es porque quieren que se lo metan. (Alumno 5o., centro B).

Hay una de octavo que es media puta y se deja manosear. Deja que los varones le agarren sus tetas y sus partes. (Alumno 60., centro A).

Las hembras son las culpables que se dejan. Hay muchas cueros, muchas putas, que después se quejan cuando los varones les hacemos algo. (Alumno 7o., centro A).

Allá en el curso de nosotros hay una muchacha que usa un pantalón y se le ve toda esta vaina. Esa provoca a todos los muchachos. Uno se vuelve loco, nosotros no tenemos la culpa. (Alumno 80., centro B).

El arraigo de los estereotipos de género en estudiantes es un resultado coherente con investigaciones anteriores, que demuestran que mientras las alumnas se sienten responsables de las situaciones de violencia, los varones tienden a minimizar o evadir responsabilidad ante los actos violentos, así como a culpabilizar a las víctimas (Díaz-Aguado, 2005; Bascón, Saavedra y Arias, 2013). 


\section{Conclusiones}

Conocer la percepción que tienen docentes y estudiantes sobre las causas de la violencia escolar es un elemento clave para el diseño e implementación de programas preventivos.

El estudio evidenció la existencia de débiles lazos de amistad entre el estudiantado y de fronteras difusas entre el juego y la violencia; asimismo, el rol que desempeñan los "enchinchadores" en la provocación de episodios de violencia escolar. Todos estos son elementos importantes que se vinculan con la interiorización de la violencia como una forma de diversión. Lo anterior, junto al arraigo a estereotipos tradicionales de género, revela una asimilación de mecanismos de desconexión moral y de desplazamiento de responsabilidad (Ortega, 2008; Ortega, Sánchez y Menesini, 2002; Sánchez, Ortega y Menesini, 2012).

Considerar el contexto familiar como causa principal de la violencia escolar sitúa al cuerpo docente al margen de las posibilidades de actuación. Además, imposibilita que la escuela reflexione sobre su rol en el ejercicio y la reproducción de la misma. En ese sentido, diversos autores recuperan el concepto violencia de la escuela para hacer explícita la manera en que la cultura adultocéntrica y la no atención a la diversidad favorecen y perpetúan la violencia escolar (Debarbieux y Blaya, 2010; Pacheco-Salazar, 2015). Por otra parte, la violencia interiorizada como una forma de diversión y el arraigo a estereotipos tradicionales de género impiden que el estudiantado visualice la necesidad de relacionarse de forma igualitaria y respetuosa con sus pares.

En los centros educativos se recrean y construyen modelos de convivencia social. Por tanto, es relevante que los programas educativos destinados a prevenir y erradicar la violencia escolar consideren el trabajo desde la educación emocional, la atención a la diversidad, y el aprendizaje y el trabajo cooperativos.

En futuros estudios, se recomienda profundizar en el rol del espectador y el provocador, así como en la percepción sobre las causas de la violencia escolar que tienen las familias y el personal administrativo de los centros educativos.

\section{Agradecimientos}

La investigación que sustenta este artículo fue realizada gracias al apoyo del Ministerio de Educación de la República Dominicana (MINERD), el Instituto Nacional de Formación y Capacitación del Magisterio (Inafocam) y el Instituto Tecnológico de Santo Domingo (INTEC).

\section{Referencias}

Abramovay, M. (2005). Violencia en las escuelas: un gran desafío. Revista Iberoamericana de Educación, 38, 53-66.

Alonso, P. (2009). La visión del fenómeno bullying en alumnos del Curso de Aptitud Pedagógica en los años 2005/06 y 2006/07: semejanzas y diferencias. Estudios Sobre Educación, 17, 125-144.

Bascón, M., Saavedra, J. y Arias, S. (2013). Conflictos y violencia de género en la adolescencia. Análisis de estrategias discursivas y recursos para la coeducación. Profesorado. Revista de Currículum y Formación de Profesorado, 17(1), 289-307.

Córdoba, F., Del Rey, R., Casas, J. y Ortega, R. (2016). Valoración del alumnado de primaria sobre convivencia escolar: El valor de la red de iguales. Psicoperspectivas, 16(2), 79-90.

Debarbieux, E. y Blaya, C. (2010). Sociología y violencia escolar: un enfoque contextual. En R. Ortega (Coord.), Agresividad injustificada, bullying y violencia escolar (pp. 355-383). Madrid: Alianza Editorial. 
Del Rey, R. y Ortega, R. (2007). Violencia escolar: claves para comprenderla y afrontarla. Escuela Abierta, 10, 77-89.

DeRosier, M. y Newcity, J. (2005). Students' perceptions of the school climate. Journal of School Violence, 4(3), 3-19.

Díaz-Aguado, M. (2005). La violencia entre iguales en la adolescencia y su prevención desde la escuela. Psicothema, 17(4), 549-558.

Díaz-Aguado, M. (2007). Pautas para la prevención de los conflictos. En M. De Esteban (Coord.), Nuevos retos para convivir en las aulas: construyendo la escuela cívica. (pp. 71-131). Madrid: Fundación Europea Sociedad y Educación.

Díaz-Aguado, M. y Martínez, R. (2014). Types of adolescent male dating violence against women, selfesteem, and justification of dominance and aggression. Journal of Interpersonal Violence, 30(15), 1-23.

Foshee, V. A., Karriker Jaffe, K. J., Reyes, H. L. M., Ennett, S. T., Suchindran, C., Bauman, K. E., \& Benefield, T. S. (2013). The peer context and the development of the perpetration of adolescent dating violence. Journal of Youth and Adolescence, 42(4), 471-486.

Fundación Santa María y oel. (2008). Estudio de convivencia escolar en la República Dominicana. Santo Domingo: Autores.

Garaigordobil, M. y Oñederra, J. A. (2010). La violencia entre iguales. Revisión teórica y estrategias de intervención. Madrid: Pirámide.

Giordano, P., Kaufman, A., Manning, W. y Longmore, M. (2015). Teen dating violence: the influence of friendships and school context. Sociological Focus, 48, 150-171.

Giraldo, M. (2011). Abordaje de la investigación cualitativa a través de la teoría fundamentada en los datos. Ingeniería Industrial. Actualidad y Nuevas Tendencias, 2(6), 79-86.

Gómez-Ortiz, O., Del Rey, R., Casas, J. A. y Ortega, R. (2014). Parenting styles and bullying Involvement. Culture and Education, 26(1), 132-158.

Gómez-Ortiz, O., Romera, E. y Ortega-Ruiz, R. (2016). Parenting styles and bullying. The mediating role of parental psychological aggression and physical punishment. Child Abuse y Neglect, 51, 132-143.

Gómez, A. (2013). Bullying: el poder de la violencia. Una perspectiva cualitativa sobre acosadores y víctimas en escuelas primarias de Colima. Revista Mexicana de Investigación Educativa, 18(58), 839-870.

Instituto Dominicano de Evaluación e Investigación Educativa y Universidad Iberoamericana. (2014). Estudio de prevalencia, tipología y causas de la violencia en los centros educativos de básica y media de la República Dominicana. Santo Domingo: Autores.

Jares, X. (2006). Conflicto y convivencia en los centros educativos de secundaria. Revista de Educación, $339,467-491$.

Kerbs, J. y Jolley, J. (2007). The Joy of violence: what about violence is fun in middle-school? American Journal of Criminal Justice, 32(1), 12-29.

Milletich, R., Kelley, M., Doane, A. y Pearson, M. (2010). Exposure to interparental violence and childhood physical and emotional abuse as related to physical aggression in undergraduate dating relationships. Journal of Family Violence, 25(7), 627-637. 
Murillo, P. y Becerra, S. (2009). Las percepciones del clima escolar por directivos, docentes y alumnado mediante el empleo de "redes semánticas naturales". Su importancia en la gestión de los centros educativos. Revista de Educación, 350, 375-399.

Ortega, R. (2008). Malos tratos entre escolares: de la investigación a la intervención. Madrid: Ministerio de Educación, Política Social y Deporte.

Ortega, R., Sánchez. V. y Menesini, E. (2002). Violencia entre iguales y desconexión moral: Un análisis transcultural. Psicothema, 14(1), 37-49.

Pacheco-Salazar, B. (2015). Reflexiones sobre la no atención a la diversidad como violencia de la escuela. Revista Ciencia y Sociedad, 40(4), 663-684.

Perrenoud, P. (2008). Diez nuevas competencias para enseñar. Tiempo de Educar, 9(17), 153-159.

Renner, L. y Whitney, S. (2012). Risk factors for unidirectional and bidirectional intimate partner violence among young adults. Child Abuse \& Neglect, 36(1), 40-52.

Restrepo-Ochoa, D. (2013). La teoría fundamentada como metodología para la integración del análisis procesual y estructural en la investigación de las representaciones sociales. Revista CES Psicología, 6(1), 122-133.

Sánchez, V., Ortega, R. y Menesini, E. (2012). La competencia emocional de agresores y víctimas de bullying. Anales de psicología, 28(1), 71-82.

Strauss, A. y Corbin, J. (2002). Bases de la investigación cualitativa. Técnicas y procedimientos para desarrollar la teoría fundamentada. Medellín: Universidad de Antioquia.

Torrego, J. C. (2006). Desde la mediación de conflictos en centros escolares hacia el modelo integrado de mejora de la convivencia. En J. C. Torrego (Coord.), Modelo integrado de mejora de la convivencia. Estrategias de mediación y tratamiento de conflictos (pp.11-26). Barcelona: Graó.

Vagi, K., Rothman, E., Latzman, N., Tharp, A., Hall, D. y Breiding, M. (2013). Beyond correlates: a review of risk and protective factors for adolescent dating violence perpetration. Journal of Youth and Adolescence, 42(4), 633-649.

Vargas, T. (2010). Violencia en la escuela. Estudio cualitativo 2008-2009. Santo Domingo: Plan Internacional.

Viguer, P. y Solé, N. (2012). Escuela e iguales como contextos de socialización en valores y convivencia: una investigación participativa a través de un debate familiar. Cultura y Educación, 24(4), 475-487.

Waldrip, A., Malcolm, K. y Jensen-Campbell, L. (2008). With a little help from your friends: the importance of high-quality friendships on early adolescent adjustment. Social Development, 17(4), 832-852. 